\title{
Mosaic structure of the penA gene in the oropharynx of men who have sex with men negative for gonorrhoea
}

International Journal of STD \& AIDS 2020, Vol. 3I(3) 230-235

(C) The Author(s) 2020 Article reuse guidelines: sagepub.com/journals-permissions DOI: 10.1 | $77 / 09564624 \mid 9889265$ journals.sagepub.com/home/std (S)AGE

\author{
Antonella Marangoni ${ }^{1, *}$, Giacomo Marziali' '*, Melissa Salvo', \\ Antonietta D'Antuono ${ }^{2}$, Valeria Gaspari ${ }^{2}$, Claudio Foschi' (1) \\ and Maria Carla $\mathbf{R e}^{\prime}$
}

\begin{abstract}
The oropharynx represents a crucial site for the emergence of multi-drug resistance in Neisseria gonorrhoeae. The mosaic penA alleles, associated with decreased susceptibility to cephalosporins, have emerged by DNA recombination with partial penA genes, particularly those from commensal pharyngeal Neisseria species. Here, we investigated the prevalence of the mosaic structure of the penA gene in the oropharynx of men who have sex with men testing negative for pharyngeal gonorrhoea. From January 2016 to June 2018, 35I gonorrhoea-negative men who have sex with men attending a sexually transmitted infection clinic in Italy were enrolled. Pharyngeal swabs underwent a real-time polymerase chain reaction (PCR) for the detection of the mosaic penA gene. In case of positivity, PCR products were sequenced and searched against several sequences of Neisseria strains. Overall, 31 patients $(8.8 \%)$ were found positive for the presence of the mosaic penA gene. The positivity was significantly associated with previous cases of pharyngeal gonorrhoea (relative risk [RR]: 3.56, 95\% confidence interval I.44-8.80) and with recent exposure to beta-lactams (RR: 4.29, $95 \%$ confidence interval 2.20-8.38). All penA-positive samples showed a high relatedness (90-99\%) with mosaicpositive Neisseria strains. Our data underline that commensal Neisseria species of the oropharynx may be a significant reservoir for genetic material conferring antimicrobial resistance in N. gonorrhoeae.
\end{abstract}

\section{Keywords}

Gonorrhoea (Neisseria gonorrhoeae), antibiotic, epidemiology, homosexual, prevention

Date received: 13 September 2019; accepted: 22 October 2019

\section{Introduction}

Sexually transmitted pharyngeal infections due to Neisseria gonorrhoeae are very common among men who have sex with men (MSM). ${ }^{1,2}$

The oropharynx represents a crucial site for the emergence of multi-drug resistance in $N$. gonorrhoeae. ${ }^{3}$ Indeed, it has been reported that the mosaic pen $A$ alleles, associated with decreased susceptibility and resistance to extended-spectrum cephalosporins, have emerged by DNA transformation and recombination with partial pen $A$ genes, particularly those from $N$. meningitidis or commensal oropharyngeal Neisseria species, such as $N$. perflava, $N$. cinerea, $N$. flavescens, $N$. polysaccharea. ${ }^{4}$

Several studies have described the detection of molecular markers associated with cephalosporin resistance directly from $N$. gonorrhoeae NAAT-positive samples, including pharyngeal specimens. ${ }^{5-7}$ Nevertheless, no exhaustive information is available regarding the presence of mosaic pen $A$ genes in gonorrhoea-negative pharyngeal samples. Since MSM are at higher risk for pharyngeal gonorrhoea transmission, the presence of the pen $A$ genes at the pharyngeal

\footnotetext{
'Microbiology, DIMES, University of Bologna, Bologna, Italy

${ }^{2}$ Dermatology, St Orsola-Malpighi Hospital, Bologna, Italy

*These authors have contributed equally to this work.

\section{Corresponding author:}

Claudio Foschi, Microbiology, DIMES, University of Bologna, St Orsola Hospital, Via Massarenti, 9, Bologna 40I38, Italy.

Email: claudio.foschi2@unibo.it
} 
site in $N$. gonorrhoeae-negative subjects could be considered a useful predictor and a potential precursor for a future acquisition of cephalosporin resistance in case of gonorrhoea infection.

In this context, the aim of this study was to investigate the prevalence of penA gene's mosaic structure in the oropharynx of MSM reporting condomless oral intercourse, who were negative for pharyngeal gonorrhoea.

\section{Methods}

\section{Study population and sample collection}

From January 2016 to June 2018, 351 consecutive MSM attending the sexually transmitted infection (STI) Outpatients Clinic of St Orsola-Malpighi Hospital in Bologna (Italy) and reporting condomless oral intercourse were enrolled for the study. Exclusion criteria were the following: being younger than 18 years and testing positive for pharyngeal $N$. gonorrhoeae.

After a preliminary interview, a clinical visit was carried out for each patient. Information about oropharyngeal symptoms, previous STIs and HIV status were collected, following the regular STI evaluation at the Clinic. ${ }^{8}$

Afterwards, a pharyngeal swab (E-Swab, Copan, Italy) for the molecular detection of Chlamydia trachomatis and $N$. gonorrhoeae was collected. To avoid biases related to antimicrobial treatment, only samples collected at the first visit were considered, excluding specimens obtained during the follow-up period.

Written consent was obtained from all the patients and the study protocol was reviewed and approved by the Ethics Committee of St Orsola-Malpighi Hospital in Bologna (Italy) (78/2017/U/Tess).

\section{Diagnosis of C. trachomatis and N. gonorrhoeae infections and detection of the penA gene}

Pharyngeal swabs were processed by the Versant CT/ GC DNA 1.0 Assay (Siemens Healthcare Diagnostics, Tarrytown, USA), a commercial NAAT simultaneously detecting the presence of $C$. trachomatis and/or $N$. gonorrhoeae DNA. ${ }^{9}$

This molecular assay has proved to be extremely sensitive in the detection of extra-genital $N$. gonorrhoeae infections (limit of detection: 1.0 copies $/ \mathrm{ml}$ ), with an excellent specificity (no false positive results due to the presence of non-gonococcal Neisseria species). ${ }^{9}$

Starting from the remaining DNA eluate of the Versant polymerase chain reaction (PCR) plate, gonorrhoea-negative samples were processed for the detection of the mosaic structure of the pen $A$ gene by a TaqMan real-time PCR, as previously described in detail by Ochiai et al. ${ }^{10}$ Specific amplification of the pen $A$ gene was performed with the primers NG89-F2 (5'-GTTGGATGCCCGTACTGGG-3') and NG89-R1 (5'-ACCGATTTTGTAAGGCAGGG-3') and with the probe NG89-P1 (5'-56-FAM-CGGCAAAGTGGAT GCAACCGA-3IABkFQ-3'). The components of PCR in a final volume of $50 \mu$ included Platinum Quantitative PCR Super Mix-UDG with ROX (Invitrogen), $1 \mu \mathrm{M}$ of the primers, $250 \mathrm{nM}$ of the probe and $5 \mathrm{mM}$ of $\mathrm{MgCl}_{2}$. Finally, $5 \mu$ l of template was added to each reaction mixture. An ABI PRISM 7300 realtime PCR system (Applied Biosystems, Japan) was used for amplification and detection, using the following cycling conditions: $2 \mathrm{~min}$ at $50^{\circ} \mathrm{C}, 10 \mathrm{~min}$ at $95^{\circ} \mathrm{C}$, and 40 cycles of $15 \mathrm{~s}$ at $95^{\circ} \mathrm{C}$ and $60 \mathrm{~s}$ at $60^{\circ} \mathrm{C}$.

\section{penA amplicon sequencing}

In case of a pen $A$-positive sample, the $220 \mathrm{bp}$ fragment PCR products were sequenced using forward and reverse primers. The pen $A$ amplicons were sequenced by the dye terminator method with an automatic sequencer. First, to confirm the specificity for Neisseriaceae, pen $A$ amplicon sequences were searched against the entire nucleotide database on the National Center for Biotechnology Information (NCBI) website, using online BLAST analysis (https://blast.ncbi.nlm. nih.gov/Blast.cgi).

Second, pen $A$ sequence data were compared using the genome comparator tool, implemented within the PubMLST.org/neisseria website. As previously described, in this website defined loci are allocated a value free nomenclature using the prefix NEIS followed by four digits. ${ }^{11}$ Penicillin binding protein 2 was defined as NEIS1753 and was associated with the locus tag NGO1542 (from the reference $N$. gonorrhoeae isolate FA1090) and the gene name, penA. ${ }^{11}$ Through this comparator tool, the allelic variant of penA gene (2131 available alleles) is therefore obtained.

Finally, to confirm the presence of penA mosaic structure, obtained electropherograms were BLAST searched against several Neisseria strains, including sequences from both mosaic-positive (accession numbers: AY294556; AB071984; AB546858) and -negative strains (CP003909; CP001050).

\section{Statistical analysis}

All statistical analyses were performed by using GraphPad Prism software (GraphPad Software, San Diego, California, USA, www.graphpad.com). To evaluate statistically significant differences, t-test was used to compare quantitative data, whereas categorical data were analysed through the calculation of 
relative risk ( $\mathrm{RR})$ and odds ratio $(\mathrm{OR})$, considering a $95 \%$ confidence interval $(\mathrm{CI})$. A $P$ value $<0.05$ was considered significant.

\section{Results}

\section{Study population}

The mean age of the 351 subjects enrolled was $33.7 \pm$ 10.3 years ( \pm standard deviation) (min-max $19-77$ years). Out of the 351 patients, $56(15.9 \%)$ complained about various symptoms, including pharyngeal pain, hoarseness and painful cervical adenopathy. Globally in the cohort of subjects enrolled, 71 patients $(71 / 351 ; 20.2 \%)$ were HIVpositive and $20(20 / 351 ; 5.7 \%)$ were positive for pharyngeal C. trachomatis infection.

\section{Prevalence of penA gene at the pharyngeal site}

Overall, 31 patients $(31 / 351 ; 8.8 \%)$ were found positive for the presence of the mosaic structure of the pen $A$ gene. The detection of pen $A$ gene was not associated with the age of patients (mean age of positive versus negative subjects: $33.6 \pm 10.8$ versus $33.7 \pm 10.2$ years; $P=0.96)$ nor with the presence of pharyngeal symptoms (RR: 0.70 with $95 \%$ CI of $0.28-1.78$; OR: 0.68 with $95 \%$ CI of $0.25-1.85$ ), nor with the HIV status (7.0\% of HIV-positive subjects versus $11.8 \%$ of HIVnegative patients; RR: 1.58 with $95 \%$ CI of $0.64-3.88$; OR: 1.67 with $95 \%$ CI of $0.60-4.65$ ).

Interestingly, analysing clinical data of patients within six months prior to enrolment, we found that the detection of penA was significantly associated with previous cases of pharyngeal gonorrhoea $(12.8 \%$ versus $3 \%$; RR: 3.56 with $95 \%$ CI of 1.44-8.80; OR: 4.59 with $95 \%$ CI of 1.34-15.63), as well as with a recent exposure to beta-lactam antibiotics (e.g. beta-lactams for genital and/or extra-genital gonorrhoea, active syphilis, pharyngeal infection due to Streptococcus pyogenes) (33.3\% versus $7.7 \%$; RR: 4.29 with $95 \%$ CI of $2.20-8.38$; OR: 5.61 with $95 \%$ CI of $2.38-13.24$ ).

\section{Sequencing of penA amplicons}

When searched against the entire nucleotide database on the NCBI website, all pen $A$ amplicon sequences always align only with Neisseria species, confirming the specificity of PCR products. Through the genome comparator tool implemented within the PubMLST. $\mathrm{org} /$ neisseria website, we found that most of pen $A$ sequences showed the closest match with pen $A$ allele $2028(18 / 31 ; 58 \%)$, followed by allele $879(11 / 31$; $35.5 \%$ ) (Table 1).

All the alignments between pen $A$ amplicon sequences and the closest penA allelic variant are shown in the Supplementary material.
As shown in Table 1, all penA-positive samples showed a high relatedness (ranging from 90 to $99 \%$ ) with several mosaic-positive Neisseria strains. At the same time, penA sequences showed no match or low relatedness $(<81 \%)$ with mosaic-negative strains.

\section{Discussion}

The possibility of acquiring cephalosporin resistance from pharyngeal commensal Neisseria species underlines the crucial role of the pharynx in the creation of 'multi-drug resistant' gonorrhoea. ${ }^{12-15}$ The detection of molecular markers associated with cephalosporin resistance in $N$. gonorrhoeae-negative pharyngeal samples in patients at higher risk for gonorrhoea (e.g. MSM) could open new perspectives for the prevention of antimicrobial resistance.

In this study, we assessed the prevalence of the mosaic structure of the pen $A$ gene in the oropharynx of 351 MSM reporting condomless oral intercourse but negative for pharyngeal gonorrhoea. For this purpose, we used a previously described real-time PCR employed both on Neisseria isolates and directly on $N$. gonorrhoeae-positive clinical samples. ${ }^{6,10}$

In this context, it should be taken into account that pen $A$ genes could be potentially fragmented by enzymes present in the pharyngeal environment, thus leading to an underestimation of pen $A$-positive samples.

First, we found that more than $8 \%$ of the subjects enrolled were positive for the presence of the pen $A$ gene and we confirmed that pen $A$ amplicons were highly related with Neisseria strains harbouring the mosaic structure of the pen $A$ gene.

It has been shown that commensal Neisseria spp. are a source of altered pen $A$ alleles and that the horizontal gene transfer is a common mechanism for beta-lactam resistance in Neisseria species. ${ }^{16}$ As example, N. subflava and $N$. cinerea may be involved in the emergence of $N$. gonorrhoeae strains with either intermediate or total resistance to penicillin or cephalosporin by the horizontal genetic exchange of the pen $A$ gene. ${ }^{14,17}$

Second, we found that pen $A$ positivity was associated with previous cases of pharyngeal gonorrhoea and with the recent exposure to beta-lactams (e.g. cephalosporins for genital and extra-genital gonorrhoea, penicillin for syphilis).

Recently, Dong et al., ${ }^{18}$ by means of a culture-based approach, showed that MSM harbour in the oropharynx a high rate of commensal Neisseria species with reduced susceptibility to cephalosporins. Moreover, they demonstrated that the recent use of antibiotics is strongly associated with the presence of antimicrobialresistant Neisseria strains. $^{18}$

These results emphasise how the use of antibiotics to treat common STIs could contribute to the onset of 
Table I. Characteristics of penA amplicon sequences. For each sequence, the closest match with penA alleles, as well as the relatedness with several mosaic-positive (AY294556,AB071984, AB546858) and -negative Neisseria strains (CP003909, CP00I050), are reported.

\begin{tabular}{|c|c|c|c|c|c|c|}
\hline \multirow{2}{*}{$\begin{array}{l}\text { penA amplicon } \\
\text { sequence }\end{array}$} & \multirow{2}{*}{$\begin{array}{l}\text { penA allele }{ }^{a} \\
\text { (NEISI } 753 \text { ) }\end{array}$} & \multicolumn{3}{|c|}{$\begin{array}{l}\text { Per cent identity after alignment } \\
\text { with mosaic-positive Neisseria strains } \\
\text { (accession numbers) }\end{array}$} & \multicolumn{2}{|c|}{$\begin{array}{l}\text { Per cent identity after alignment } \\
\text { with mosaic-negative Neisseria strains } \\
\text { (accession numbers) }\end{array}$} \\
\hline & & AY294556 & AB07। 984 & AB546858 & СР003909 & СР00I050 \\
\hline 이 & 2068 & 95.1 & 95.1 & 90.6 & 80.5 & 80.5 \\
\hline 02 & 2068 & 96.1 & 96.1 & 91.4 & 80.5 & 80.5 \\
\hline 03 & 2068 & 95.5 & 95.5 & 90.8 & 80.0 & 80.0 \\
\hline 04 & 2068 & 94.9 & 94.9 & 90.1 & 80.6 & 80.6 \\
\hline 05 & 2068 & 95.5 & 95.5 & 90.8 & 80.0 & 80.0 \\
\hline 06 & 879 & 96.7 & 98.9 & 94.5 & 0.0 & 0.0 \\
\hline 07 & 879 & 96.2 & 98.3 & 93.9 & 0.0 & 0.0 \\
\hline 08 & 879 & 97.2 & 99.4 & 94.9 & 0.0 & 0.0 \\
\hline 09 & 879 & 96.2 & 98.3 & 93.9 & 0.0 & 0.0 \\
\hline 10 & 931 & 93.2 & 95.2 & 93.8 & 0.0 & 0.0 \\
\hline II & 879 & 96.3 & 98.4 & 97.2 & 0.0 & 0.0 \\
\hline 12 & 2068 & 94.7 & 94.7 & 93.8 & 80.6 & 80.6 \\
\hline 13 & 2068 & 96.1 & 96.1 & 93.8 & 80.6 & 80.6 \\
\hline 14 & 2068 & 95.2 & 95.2 & 93.4 & 80.7 & 80.7 \\
\hline 15 & 2068 & 94.9 & 94.9 & 90.3 & 79.4 & 79.4 \\
\hline 16 & 2068 & 94.3 & 94.3 & 90.7 & 80.0 & 80.0 \\
\hline 17 & 879 & 96.2 & 98.3 & 93.9 & 0.0 & 0.0 \\
\hline 18 & 2068 & 95.1 & 95.1 & 90.6 & 80.5 & 80.5 \\
\hline 19 & 879 & 96.2 & 98.3 & 96.6 & 0.0 & 0.0 \\
\hline 20 & 2068 & 95.4 & 95.4 & 90.8 & 79.9 & 79.9 \\
\hline 21 & 2068 & 94.9 & 94.9 & 90.3 & 79.8 & 79.8 \\
\hline 22 & 2068 & 95.1 & 95.1 & 90.6 & 80.5 & 80.5 \\
\hline 23 & 879 & 96.1 & 98.3 & 93.9 & 0.0 & 0.0 \\
\hline 24 & 2068 & 95.4 & 95.4 & 90.8 & 79.8 & 79.8 \\
\hline 25 & 2068 & 95.1 & 95.1 & 90.6 & 80.5 & 80.5 \\
\hline 26 & 2068 & 94.2 & 94.2 & 90.0 & 79.7 & 79.7 \\
\hline 27 & 931 & 92.6 & 94.7 & 93.3 & 0.0 & 0.0 \\
\hline 28 & 879 & 96.7 & 98.9 & 94.5 & 0.0 & 0.0 \\
\hline 29 & 2068 & 96.1 & 96.1 & 91.4 & 80.5 & 80.5 \\
\hline 30 & 879 & 95.8 & 97.9 & 96.6 & 0.0 & 0.0 \\
\hline 31 & 879 & 96.2 & 98.3 & 93.9 & 0.0 & 0.0 \\
\hline
\end{tabular}

${ }^{a}$ penA sequences were compared using the genome comparator tool, implemented within the PubMLST.org/neisseria website. Penicillin binding protein 2 locus is defined as NEISI753 and is associated with the locus tag NGOI542 (from the reference N. gonorrhoeae isolate FAI090).

antimicrobial resistance in commensal Neisseria species of the oropharynx. The prevention of bacterial STIs is therefore a crucial component to fight the hard challenge of drug resistance.

In this context, modelling work by Fingerhuth et al. $^{19}$ demonstrated that higher treatment rates result in faster spread of antibiotic resistance in $N$. gonorrhoeae. We should probably revise the current notion that more screening and treatment will limit the spread of $N$. gonorrhoeae, perhaps considering not to screen and treat asymptomatic pharyngeal gonorrhoea. Treatment recommendations for $N$. gonorrhoeae should therefore carefully balance prevention of infection and avoidance of resistance spread.

Our molecular-based approach does not allow us to identify the particular Neisseria species harbouring the pen $A$ mosaic structure. In addition, no detailed information about the susceptibility/resistance to the various cephalosporins can be obtained. However, unlike culture, our approach is easier and faster and it can be successfully combined with a molecular assay for STI screening of the oropharynx.

We are fully aware of a significant limitation of this study: the entire pen $A$ gene should be sequenced to 
obtain a detailed molecular analysis of the mosaic structure. In this way, it would be possible to evaluate the association with specific bacterial strains, as well as the presence of clusters within the population enrolled.

In conclusion, even though preliminary, our data underline that commensal Neisseria species of the oropharynx may be a significant reservoir for genetic material conferring antimicrobial resistance in $N$. gonorrhoeae. For this reason, to prevent the spread of antimicrobial resistance in $N$. gonorrhoeae, individuals should be advised on correct and consistent use of the condom even during oral intercourse to minimise the potential transmission of resistance genes.

Longitudinal studies, including also the heterosexual population, will be needed to investigate the relationship between the previous pharyngeal detection of penA-positive Neisseria species and the future acquisition of mosaic penA-positive $N$. gonorrhoeae.

\section{Acknowledgements}

We would like to thank Giorgia Adami, Roberta Licciardi and Marielle Ezekielle Djusse for providing excellent technical support during the study. Moreover, we are grateful to Matteo Belletti for his excellent and skilful assistance during the patients' enrolment.

\section{Declaration of conflicting interests}

The authors declared no potential conflicts of interest with respect to the research, authorship, and/or publication of this article.

\section{Funding}

The authors disclosed receipt of the following financial support for the research, authorship, and/or publication of this article: This study was supported by the Ministry of Education, University and Research (MIUR), Italy (RFO 2015_2016 to AM). The funder had no role in study design, data collection and analysis, decision to publish, or preparation of the article.

\section{ORCID iD}

Claudio Foschi (D) https://orcid.org/0000-0001-7253-8799

\section{References}

1. Chan PA, Robinette A, Montgomery M, et al. Extragenital infections caused by Chlamydia trachomatis and Neisseria gonorrhoeae: a review of the literature. Infect Dis Obstet Gynecol 2016; 2016: 5758387.

2. Gaspari V, Marangoni A, D'Antuono A, et al. Pharyngeal chlamydia and gonorrhea: a hidden problem. Int J STD AIDS 2019; 30: 732-738.
3. Unemo $\mathrm{M}$ and Shafer WM. Antimicrobial resistance in Neisseria gonorrhoeae in the 21st century: past, evolution, and future. Clin Microbiol Rev 2014; 27: 587-613.

4. Osaka K, Takakura T, Narukawa K, et al. Analysis of amino acid sequences of penicillin-binding protein 2 in clinical isolates of Neisseria gonorrhoeae with reduced susceptibility to cefixime and ceftriaxone. $J$ Infect Chemother 2008; 14: 195-203.

5. Whiley DM, Trembizki E, Buckley C, et al. Molecular antimicrobial resistance surveillance for Neisseria gonorrhoeae, Northern Territory, Australia. Emerg Infect Dis 2017; 23: 1478-1485.

6. Nicol M, Whiley D, Nulsen M, et al. Direct detection of markers associated with Neisseria gonorrhoeae antimicrobial resistance in New Zealand using residual DNA from the Cobas $4800 \mathrm{CT} / \mathrm{NG}$ NAAT assay. Sex Transm Infect 2015; 91: 91-93.

7. Donà V, Smid JH, Kasraian S, et al. Mismatch amplification mutation assay-based real-time PCR for rapid detection of Neisseria gonorrhoeae and antimicrobial resistance determinants in clinical specimens. $J$ Clin Microbiol 2018; 56: pii:e00365-18.

8. Foschi C, Gaspari V, Sgubbi P, et al. Sexually transmitted rectal infections in a cohort of 'men having sex with men'. J Med Microbiol 2018; 67: 1050-1057.

9. Marangoni A, Foschi C, Nardini P, et al. Evaluation of the versant CT/GC DNA 1.0 assay (kPCR) for the detection of extra-genital Chlamydia trachomatis and Neisseria gonorrhoeae infections. PLoS One 2015; 10: e0120979.

10. Ochiai S, Ishiko H, Yasuda $\mathrm{M}$, et al. Rapid detection of the mosaic structure of the Neisseria gonorrhoeae penA Gene, which is associated with decreased susceptibilities to oral cephalosporins. J Clin Microbiol 2008; 46: 1804-1810.

11. Harrison OB, Clemence M, Dillard JP, et al. Genomic analyses of Neisseria gonorrhoeae reveal an association of the gonococcal genetic island with antimicrobial resistance. J Infect 2016; 73: 578-587.

12. Spratt BG, Bowler LD, Zhang QY, et al. Role of interspecies transfer of chromosomal genes in the evolution of penicillin resistance in pathogenic and commensal Neisseria species. J Mol Evol 1992; 34: 115-125.

13. Unemo M. Current and future antimicrobial treatment of gonorrhoea - the rapidly evolving Neisseria gonorrhoeae continues to challenge. BMC Infect Dis 2015; 15: 364.

14. Igawa G, Yamagishi Y, Lee KI, et al. Neisseria cinerea with high ceftriaxone MIC is a source of ceftriaxone and cefixime resistance-mediating penA sequences in Neisseria gonorrhoeae. Antimicrob Agents Chemother 2018; 62: pii:e02069-17.

15. Ohnishi M, Watanabe Y, Ono E, et al. Spread of a chromosomal cefixime-resistant penA gene among different Neisseria gonorrhoeae lineages. Antimicrob Agents Chemother 2010; 54: 1060-1067.

16. Karch A, Vogel U and Claus H. Role of penA polymorphisms for penicillin susceptibility in Neisseria lactamica 
and Neisseria meningitidis. Int J Med Microbiol 2015; 305 : 729-735.

17. Furuya R, Onoye Y, Kanayama A, et al. Antimicrobial resistance in clinical isolates of Neisseria subflava from the oral cavities of a Japanese population. J Infect Chemother 2007; 13: 302-304.

18. Dong HV, Pham LQ, Nguyen HT, et al. Decreased cephalosporin susceptibility of oropharyngeal Neisseria species in antibiotic-using men-who-have-sex-with-men of Hanoi, Vietnam. Clin Infect Dis 2019; pii:ciz365.

19. Fingerhuth SM, Bonhoeffer S, Low N, et al. Antibioticresistant Neisseria gonorrhoeae spread faster with more treatment, not more sexual partners. PLoS Pathog 2016; 12: e1005611. 